\title{
Redefinición de lo educativo en el cibercontexto: a propósito de las TIC como herramienta de inclusión.
}

\author{
Enric Prats \\ enricprats@ub.edu \\ Universidad de Barcelona
}

\section{1.}

Habrá que empezar recordando que el ciberespacio no deja de ser una especie de alucinación colectiva, una recreación humana (un artefacto, en suma) que quiere imitar lo natural para transgredirlo y superarlo: «el ciberespacio es un paisaje cultural donde los ríos pueden fluir hacia arriba y los bosques pueden ser hechos de árboles cristalinos (o cosas infinitamente más extrañas)» (Mizrach, s.d.). Una metáfora para explicarnos que en ese espacio no funcionan las leyes de la física pero en el que, sin duda, puede dibujarse un paisaje propio; un paisaje cultural, artificioso, pero paisaje en definitiva [1] .

Apostar por artefactos nuevos que, supuestamente, mejoran o aumentan el bienestar individual y colectivo es una constante que viene paralela al grado de nerviosismo que esos mismos ingenios despiertan. El debate entre tecnoutópicos y tecnófobos es tan antiguo como la misma humanidad y la aparición de una nueva tecnología siempre ha sido recibida con una ambivalencia al menos curiosa: se alaban las prestaciones originales que aporta y se duda precisamente de que esas nuevas prestaciones sean del todo beneficiosas para el género humano.

En este terreno, los tecnoutópicos son capaces de rellenar esa alucinación con diversidad de argumentos, una alucinación que puede entenderse en su sentido más lato, como engaño o deslumbramiento, pero también en su sentido más estrecho, de deformación de la realidad, por una ingesta inapropiada, argumento utilizado por los tecnófobos.

En este trabajo se quiere revisar el espacio que debe ocupar la escuela en este entramado que parece vislumbrarse con la cibercultura. Si la escuela era un lugar de encuentro con el conocimiento, cuando ese encuentro es protagonizado por otros agentes hay que plantear la esencia de lo escolar y, quizás, de lo educativo. Se puede discutir precisamente acerca del sentido educativo de muchas iniciativas dirigidas a jóvenes, algo que no haremos aquí en profundidad; de hecho, en muchas reflexiones y prácticas educativas que aspiran a ser incluyentes, orientadas sobre todo a proporcionar herramientas de acceso al ciberconocimiento, tanto en el sentido más tecnológico pero también ético-cognitivo, pueden esconderse algo así como retrovalores morales, es decir valores aparentemente positivos que, desarrollados sin las debidas precauciones pedagógicas, llegan a generar efectos imprevistos e incluso contrarios a los esperados en el ámbito de la moralidad del sujeto.

Así, son frecuentes las iniciativas volcadas en proporcionar un mejor acceso a la Red por parte de fabricantes y distribuidores. Por poner algún ejemplo, Cisco Systems relata en su sitio web diversas prácticas “de éxito" llevadas a cabo para implementar redes inalámbricas en campus universitarios [2] , como el del Colegio Internacional SEK, en Chile, donde además de esa instalación se suministraron ordenadores portátiles a profesorado, con los siguientes objetivos: "1. Acceso a la información en todo el campus, movilidad; 2. Se plantea una nueva forma de enseñar apoyada en la tecnología. 3. En las clases se cuenta con más herramientas gracias a la incorporación de información visual y auditiva”.

Lo discutible de estas y otras iniciativas del mismo carácter es su misma continuidad y sostenibilidad; la dependencia técnica y por supuesto programática que generan, en el sentido de que en cierta manera “obligan” al usuario a acostumbrarse a un entorno de trabajo específico, no plantea precisamente una perspectiva de flexibilidad y adaptabilidad por parte del usuario. La fidelización en la red puede generar 
más problemas que ventajas si no se dan determinadas condiciones que faciliten un uso crítico y comprometido.

Es pues en el ámbito de los valores donde quizás encontramos un reflejo más claro de esa dualidad de emociones que genera la tecnología nueva, más relacionada con la comunicación que con la gestión en si de la información. El medio es motivo de acercamiento, para los primeros, mientras que ese acercamiento, para los segundos, es visto precisamente como fuente de conflictos, sobre todo en lo moral. Pero este punto de fricción no será original de la nueva tecnología.

En lo estrictamente educativo, Noguera nos recuerda que hablar de valores en Internet significa, al menos, dos cosas: a) analizar cómo influye esta herramienta en los usuarios jóvenes (ampliable por supuesto a usuarios menos jóvenes); b) elaborar propuestas acerca de su uso para fomentar o desarrollar determinados valores (Noguera, 2004).

El primer objetivo parece difícil de alcanzar en las actuales circunstancias, por cuanto, i) hay que reconocer que Internet llega precisamente a aquellos jóvenes "ya" conectados, es decir, que tienen otros muchos canales para acceder al conocimiento, y que en consecuencia complica cualquier análisis para discernir el peso real de la red en la conformación de determinados valores; ii) existen serias dificultades metodológicas para diagnosticar el impacto de toda nueva herramienta, que precisamente por su novedad y el corto recorrido realizado impiden completar un diagnóstico completo de la situación y exigirían estudios longitudinales de alto coste.

Por supuesto, se relaciona muy deprisa la relación existente entre ciberconexión e inclusión y entre inclusión y ejercicio de la ciudadanía. En esta línea, y precisando algo más, Eduteka [3] propone cinco valores que las TIC pueden ayudar a desarrollar en el capítulo de la educación para una ciudadanía democrática (Eduteka, 2004):

$\S \quad$ honestidad, en el sentido de evitar el plagio en la captura y reproducción de información en el momento de realizar trabajos escolares de investigación;

$\S$ solidaridad y generosidad, orientadas al desempeño colectivo en grupos de trabajo, principalmente, compartiendo conocimiento;

$\S \quad$ tolerancia y respeto, demostrando apertura mental a ideas distintas y diversas, tanto en el grupo de iguales como en los contactos cibernéticos;

$\S \quad$ responsabilidad, dirigida tanto al mantenimiento y cuidado de los equipos y bienes comunes como al uso adecuado de las TIC para los objetivos escolares y no para otros fines;

$\S \quad$ perseverancia, entendida más como una facultad que como un valor, para evitar el desfallecimiento y estimular el esfuerzo en el proceso de aprendizaje.

Se da la circunstancia que los mencionados valores configuran la columna vertebral del uso de la red, resumibles en dos valores, como son la confianza y la sobriedad, que no destacan exactamente en otros ámbitos de la vida. Educando personas confiadas y confiables, y también comedidas en sus gustos, aficiones y comportamientos conseguiremos, nada menos, que individuos perfectamente autorregulados y, a buen seguro sabe, ciudadanos responsables.

Así, la responsabilidad se erige como el núcleo de toda propuesta educativa de desarrollo de ciberhabilidades. En el mismo sentido, en el terreno de los profesionales que gestionan datos (documentalistas y archiveros en su sentido más amplio, es decir, personal de enfermería, de compañías de seguros, de agencias de viajes, empleados de banca, etc.), se supone que la responsabilidad deontológica se concreta en unos determinados patrones éticos que hay que tener presentes en todo el proceso de tratamiento de datos, desde la captura a su difusión, pasando por el almacenaje y el análisis [4] . No hay que olvidar que la circulación de esos datos por la Red se produce en unas condiciones de seguridad y fiabilidad más que discutibles, por lo que todas las precauciones son pocas.

Así, el acceso y manejo de la red se basa no tan sólo en el despliegue de unas determinadas estrategias o capacidades técnico-instructivas, sino principalmente en la aplicación de un mapa de valores 
perfectamente definido que permite ese abordaje consciente y responsable de qué hablamos.

Es dudoso, por lo tanto, que el acceso per se suponga un beneficio a corto o medio plazo para el usuario; lo será en la medida en que sea capaz de modificar el punto de anclaje de sus creencias y opiniones acerca de cómo se construye el conocimiento. De hecho, si hasta hoy era acertado pensar en términos de currículo agregado, siguiendo a Bernstein, probablemente lo que propone el aprendizaje en línea es la necesidad de un diseño integrado del currículo que abogue por un uso racionalmente crítico, algo que con estadísticas resulta difícil de defender.

Muchas son las ventajas que sin duda ofrece el nuevo medio y, paralelamente, muchos son los riesgos que se apuntan. Las valoraciones positivas de lo que significa el nuevo medio, en términos de valores, se balancean con aquellas que se sitúan en un plano apocalíptico. Lejos de la Arcadia de Virgilio, en un clima inocente y sin artificios, Internet sublima para muchos el mito de Babel, donde los humanos que quieren alcanzar el cielo cibernético son confundidos con la diversidad tecnolingüística.

Esa metáfora de la confusión y la dispersión se expande sobre todo en aquellos que se consideran excluidos del medio: la ruptura digital lo es más en términos de desplazamiento que de rechazo. Hoy por hoy, sentirse desplazado por el empuje de lo tecnológico tiene más efectos nocivos que la misma exclusión; de hecho, Internet no excluye a nadie sino todo lo contrario: a partir del momento en que potencialmente adquirimos la condición de emisores, aumenta nuestra autoestima y nos sentimos protagonistas y plenamente integrados en este cibermundo; pero cuando, contrariamente, no ejercemos tal posibilidad, nos sentimos realmente fuera de ese espacio virtual.

De la misma manera que la televisión significó un acercamiento mutuo entre los humanos porque nos "obligaba" a compartir los mismos referentes, la red permite compartir la información y además en tiempo real. Siguiendo la comparación con lo audiovisual, si la multiplicación de canales televisivos refuerza la falsa idea de que el sistema también piensa en los diferentes, ya que la multiplicación significa más de lo mismo y no diversidad y mucho menos pluralidad, el argumento de la facilidad de uso de la red permite desarrollar una trampa de democratización y universalidad del conocimiento.

Se dice que la alfabetización digital supone un nuevo reto para educadores, tanto o más que para los industriales del ramo, que sin usuarios semi-expertos no pueden colocar sus productos. En efecto, uno de los retos de cualquier nuevo medio tecnológico es formar a sus clientes potenciales en el terreno estrictamente técnico y con un lenguaje nuevo. Poseer y dominar ese argot y sobre todo estar al día de las innovaciones que van surgiendo define el nivel mínimo de esa alfabetización [5] ; en su nivel más alto, sin embargo, significa un dominio sobre la sutilezas del medio, abarcando tanto la comprensión crítica acerca de los contenidos como el desarrollo de la toma de conciencia de lo que significa el nuevo medio en la vida de las personas.

Se dan dos argumentos centrales en el desarrollo de propuestas relacionadas con el uso educativo de Internet, pero que despiertan una cierta ambivalencia: el acceso a la información y el impulso de la diversidad.

Se propone, y debe de ser cierto, que el aprendizaje con el uso de Internet es más motivador y conecta mejor con las necesidades de ese aprendiz. Además, el acceso mismo se considera condición imprescindible para moverse por el mundo actual. Un artículo que se precie, hoy, debe añadir referencias cibernéticas para ser completo puesto que una buena parte del conocimiento científico está siendo volcado en la red, casi de manera exclusiva: algunas revistas ya han abandonado la galaxia Gutenberg para siempre y se han apuntado a la cibergalaxia. Por lo demás, la tecnología permite convertir a cualquier lector en autor mismo.

Muchas son las propuestas educativas de acceder al entorno cibernético para encontrar una determinada información. En este caso, como se sabe, el acceso a un gran número de información llega a ser abrumador y, cuando se realiza con poco criterio, las dificultades de ese acceso desbocado se disparan, con lo que aumenta en la misma proporción la vulnerabilidad del sujeto. 
Veamos un ejemplo con algo tan delicado como la salud; cuando el usuario decide recurrir a Internet para encontrar información sanitaria o biomédica importante, puede encontrarse con cuatro tipos de dificultades (Vivas; Armayones, et al., 2004) [6] :

$\S \quad$ abundancia y heterogeneidad de la información volcada en la red (en el caso que nos ocupa: diagnósticos, etiologías, terapias, fármacos, etc.);

$\S$ incomprensión de la información encontrada, que aumenta con la dificultad de legibilidad de dicha información, correlativo al grado de instrucción del usuario;

$\S$ dudas sobre la validez de las informaciones en línea, debido a la falta de regulación de los servicios que aportan la información y a investigaciones sobre la misma validez de esas informaciones, lo cual repercute en el descontento por la eficacia de la información encontrada;

$\S$ ausencia de cobertura legal, debido a la dificultad conocida de controlar los contenidos en la red.

El mismo documento recoge las conclusiones de un estudio (Berland et al., 2001) donde se concluye que las informaciones sobre salud en la red son poco fiables, y más aún en castellano que en inglés. Sin embargo, el problema no se origina con Internet sino que proviene de una dificultad inherente a la misma definición de calidad. Otro estudio citado por la misma fuente concluye que debe darse una prioridad absoluta en definir lo que se entiende por calidad, en establecer parámetros de calidad y en operativizar estos parámetros. Esta tarea se supone urgente para evitar errores de autodiagnóstico y automedicación, errores que se multiplican debido a la rapidez con que se genera, reproduce y desaparece la información en la red.

Podemos imaginar, sin datos disponibles, que la información relativa a la salud que los jóvenes y las jóvenes buscan y encuentran en la red, sobre temas de sexualidad, drogas o nutrición, alberga muchas deficiencias tanto en su fiabilidad como en su orientación ética, lo cual no hace más que incrementar el grado de vulnerabilidad de esos usuarios.

Por otro lado, el argumento central del uso escolar de Internet es la apertura a nuevos horizontes, si bien es cierto que ese proceso se propone a menudo desde lo local, después de haber asegurado un determinado grado de arraigo en lo propio. La necesidad de desarrollar unos mínimos de autoestima se sugiere como la plataforma a partir de la cual el sujeto abordará con ciertas garantías el acceso a la red.

Se asume que la diversidad es estimulante en si misma y que la posibilidad que ofrece la tecnología nueva de convertirnos en emisores y receptores de información [que no de conocimiento] genera un sentimiento de aprecio hacia las aportaciones de todo el mundo elevándolas a una categoría superior en grado de aquellas aportaciones que en el universo de la imprenta procedían del mundo de los expertos.

En otras palabras, la supuesta democratización del conocimiento significa un nuevo paso hacia la igualdad de oportunidades y sólo eso ya significa un avance sustancial en la relación con los otros [7]. Por ejemplo, los weblogs, que han pasado ya por tres generaciones, significan una apuesta auténtica para convertir Internet en un espacio de construcción compartida de conocimiento.

La apuesta por el contacto y la intercomunicación debe estudiarse desde el contenido mismo de lo que se comunica, tanto en contenido como en forma. Yus afirma que pocas veces comunicamos de manera literal, sino que "dejamos implícita toda aquella información que prevemos que nuestro interlocutor podrá obtener por sí mismo, por lo que la tarea de acceder a la interpretación pretendida por el emisor puede llegar a ser un proceso inferencial bastante complicado” (Yus, 2001).

Para corroborar lo dicho no hace falta más que acercarse a un diálogo en cualquier chat y comprobar que, entre usuarios desconocidos, la falta de referentes comunes (relativos a un espacio compartido, a un paisaje, a unos acontecimientos, por ejemplo) dificulta la dimensión pragmática de la comunicación; en concreto, el internauta reacciona compensando esa falta de información aportando datos relativos a tres ámbitos: el cuerpo físico (algo intangible todavía a través de la red), el soporte fónico (tonos y matices de voz, e incluso afonías) y lo gestual.

Para Yus, "los usuarios de Internet escriben los enunciados que les gustaría estar diciendo y leen los 
enunciados que les gustaría estar oyendo”, lo que significa que se puede recurrir con relativa facilidad, por ejemplo, a la fantasía e incluso a la mentira como apoyo argumental. Esa, seguramente, es una capacidad humana no precisamente novedosa y que no necesita ningún tipo de institucionalización educativa para ser desarrollada.

Pero lo que realmente pone de manifiesto este lenguaje nuevo, en forma y en contenido, es el riesgo de exclusión que sugiere para los no iniciados. El argumento inicial relativo a la necesidad de contacto con lo diverso puede verse negado de raíz cuando se utiliza un código restringido de comunicación, cuyas consecuencias ya anunciaba Bernstein. Además, cuando el currículo formal admite este tipo de códigos, lo que se refuerza es una sensación de pertenencia a un colectivo selecto, por supuesto, pero también se anula la posibilidad de acceder a códigos compartidos o universales, que permiten una comunicación con sectores más amplios. Restringir el formato de la comunicación a un código único, con emoticones, nuevas ortografías e incluso motes de nuevo cuño, añade incertidumbre al acto comunicativo y dificulta la comprensión del mensaje, algo que aumenta el riesgo de vulnerabilidad.

Pero la pregunta de fondo todavía no ha salido: ¿de verdad se puede hablar de construcción multicultural del saber?. García Canclini recuerda que la modernización ha impuesto que la población de las sociedades periféricas deba aprender a articular "sus" saberes tradicionales con los requerimientos técnicos y científicos propios del proceso de occidentalización algo que europeos y norteamericanos pueden ahorrarse. Volviendo al tema de la salud: «Desde una concepción evolucionista podría verse como paradójico que instrumentos como la televisión e Internet contribuyan a la expansión de medicinas tradicionales» (2004, p. 183), sobre todo, añado, cuando determinadas prácticas se presentan contradictorias con la medicina alopática y con los procedimientos del método científico.

Por lo tanto, esa co-construcción o construcción intercultural del conocimiento se reduce a los aspectos más folklóricos del saber humano, pero para nada inciden en el meollo de lo que hoy mueve el mundo. El monolingüismo imperante en la ciencia y en los negocios, a decir de García Canclini, significa que el universo de valores y creencias se está acercando a su punto de saturación, lo cual facilita su desarrollo e implantación a escala planetaria, pero deja fácilmente por el camino a los que no comparten el código y el medio. En un nivel corporativista más cercano, no hará falta recordar que para la evaluación del profesorado universitario español se valora más la publicación en revistas extranjeras que en las nacionales, algo que dice muy poco tanto de unos como de las otras.

Sin duda, el conocimiento se está globalizando y llega a sectores de población hasta ahora inmunes, pero lo discutible es la orientación de ese conocimiento. Los jóvenes de diversos lugares que interactúan en la red se encuentran hoy con más similitudes que antaño, pero esas similitudes se construyen no tanto desde la diversidad como desde la homogeneización, no tanto desde el pluralismo como desde el uniformismo. La publicidad ayuda más a conocer el mundo que la misma universidad (García Canclini, 2004, p.172), en el mismo sentido que hoy las telenovelas catalizan las emociones como antaño las novelas. Pero esa publicidad no se interesa ya por colocar sus productos, sino que vende estilos de vida, que determinadas marcas encarnan a la perfección. Terminando con García Canclini, lo que está en juego en un partido de fútbol no es la identidad deportiva de los clubes en liza o que ganen Zidane, Figo o Ronaldinho, sino que Nike coloque más publicidad que Adidas.

\section{3}

En un estudio comparativo, Castells y Díaz señalaban que el crecimiento de hogares conectados a Internet en España en el quinquenio 1996-2001 fue de casi un 2.500\% , dato que permitía prever que para el 2005 "la mitad de la población de 14 años o más estaría conectada a Internet” (Castells; Díaz, 2001, p. 3).Se recogían, entre otros muchos datos, que casi dos tercios de los hogares conectados tenían entre 3 y 5 miembros, que también casi el $60 \%$ de las personas que se conectaban tenían menos de 34 años, y que más de la mitad tenían nivel universitario, lo cual proporciona una semblanza del paralelismo entre fractura digital, fractura generacional y fractura educacional [8] .

A estas tres fracturas habría que añadir la fractura económica. Como es conocido, la incidencia de Internet en países industrializados supera en mucho a la de los países en vías de desarrollo; en 2001, según la Unión Internacional de Telecomunicaciones, la proporción de conexiones a Internet era de 12 a 1 a favor de los primeros, mientras que la de conexiones telefónicas "sólo” era de 7 a 1 (Unicef, 2004). El 
diferencial aumenta de manera geométrica con las nuevas tecnologías. A falta de datos sobre la incidencia de la telefonía móvil en países pobres, se detecta que cuando se iba produciendo un acercamiento lento pero relativamente pronunciado entre los dos grupos de países, la irrupción de Internet rompe de nuevo la expectativa de reducción de desigualdades [9] . Esta fractura económica se refleja también en el terreno demográfico cuando observamos que el $78 \%$ de la población de países industrializados vive en zonas urbanas contra el $41 \%$ de la de los países en desarrollo.

La importancia del hogar como referente de estudio para analizar la incidencia del ciberconocimiento en la vida cotidiana es crucial. En México, el 77\% de los jóvenes cuentan con televisión gratuita en su hogar, mientras que sólo el 6\% dispone de conexión a la red (García Canclini, 2004, p. 169). En otras palabras, un determinado número de jóvenes, no sólo en países menos ricos sino también en el primer mundo, construyen su conocimiento a través de «la televisión gratuita y redes informales de servicios y bienes», mediante las cuales adquieren música y cine piratas por la dificultad económica de bajarse el material de la red.

"La web del estudiante", ofrecida por el CNICE, del MEC, muestra algunos datos relativos al uso de Internet por parte de los jóvenes españoles, sin especificar la edad de referencia: el $28 \%$ de los menores visita páginas para adultos; el 38\% lo hace a páginas violentas; el 30\% ha facilitado alguna vez su número de teléfono a través de la red, o el 14,5\% ha concertado alguna cita por Internet [10] . Después de un alegato sobre las ventajas del medio, el sitio explica los "peligros", del todo ciertos sin duda, que esperan a un internauta cándido o ingenuo, principalmente los relacionados con contactos directos indeseables.

En esa preocupación por proteger más allá del contenido que se pueda visualizar en la pantalla parece darse una contradicción profunda, parejas a las que se dan en las políticas públicas dirigidas a la juventud: por un lado, se pontifica acerca de la necesidad de buscar espacios de participación con el venerable objetivo de profundizar en la inclusión y en la democracia real; por otro lado, se fomentan, por ejemplo, políticas de empleo basadas en el individualismo y la competitividad (Conde, H.; Muñoz, J.M; 2002). Estos ejes fundamentan los argumentos para el uso de Internet, algo que insistimos puede resultar contradictorio.

La fractura económica, quizás más que la generacional y la educacional, supone uno de los retos más acuciantes en este entramado que se dibuja con una cibersociedad cada vez más interconectada, donde se resaltan hasta el aburrimiento las diferencias más insólitas que esconden, sin duda, estructuras de desigualdad difíciles de superar.

Algunas organizaciones políticas juveniles plantean de manera decidida determinadas medidas para evitar una profundización en la ruptura digital y reducir los riesgos de exclusión derivados de la falta de acceso, unas medidas que se dirigen más al contacto en si que a la calidad de ese contacto. Entre dicha medidas, suelen destacarse las siguientes [11] :

$\S$ declarar Internet como espacio público, con acceso gratuito en todas las modalidades de banda, así como desarrollar y potenciar el software libre;

$\S$ proporcionar terminales de acceso a la población más desfavorecida;

$\S$ reforzar los mecanismos jurídicos que garanticen la privacidad de datos;

$\S \quad$ incrementar los recursos para formar adecuadamente a jóvenes y educadores, tanto en capacitación técnica como en contenidos digitales;

$\S \quad$ favorecer el uso de las TIC en asociaciones y entidades de jóvenes o que trabajan con jóvenes [12] , incrementando las ayudas para dotaciones;

El origen militar de Internet no debería hacernos olvidar que la necesidad de encontrar mecanismos infalibles de conexión está reñido, o al menos se encuentra en la frontera, con un conceptoamplio de libertad [13] . Este es un desafío que la escuela debe asumir como el primero. 
La vulnerabilidad de un sistema se mide en relación con su grado de apertura: a más contactos, más fragilidad. En otras palabras, en rigor, sólo son vulnerables los sistemas abiertos, de manera que lo infranqueable resulta, por definición, más seguro ante las inclemencias. El cibernauta es vulnerable por decisión propia y esa vulnerabilidad es directamente proporcional al déficit en determinadas competencias o capacidades tanto de relación social como de cognición en lo moral.

Lo que distingue a las generaciones juveniles actuales de otras anteriores no son tan sólo la cantidad de estímulos o inputs que reciben a lo largo del día para recordarles su doble condición de consumidores y objeto de consumo (así, en abstracto), sino sobre todo las condiciones por las cuales se convierten en potenciales fracasados escolares y fracasados laborales. Nunca hasta ahora se habían dado las circunstancias que permitían universalizar y democratizar auténticamente el conocimiento; tampoco hasta ahora se habían dado los niveles de bienestar y salud absoluta en la población, especialmente la más joven (mídanse niveles de estatura, por ejemplo, de una y otra generación), y tampoco se daban los requisitos para alcanzar grados de interacción tan altos entre poblaciones distantes en lo físico y en lo "químico [14] ”. Dadas estas circunstancias, las preguntas acerca del aumento relativo del fracaso escolar y de la precariedad laboral nos informa sobre un fracaso social de más calado: las sociedades que priorizan la provisionalidad por la estabilidad se arriesgan a convertir también en efímero el mapa de valores por el que se rigen y, lo que es peor, a declararse incompetentes para rejuvenecerse.

Por lo visto los jóvenes participan más en causas que en organizaciones (Reguillo, citada por García Canclini; 2004, p.177). Los sarpullidos que generan los movimientos organizados alejan la espontaneidad y el altruismo; es más atractivo y, sobre todo, "más” provisional actuar aquí y ahora que comprometerse a medio o largo plazo. Internet es el medio ideal y el más eficaz hasta el momento, junto con la telefonía móvil, para convocar y desconvocar actos: fiestas, llamamientos globafóbicos, etc.

El uso de Internet como herramienta de no-exclusión, o al menos de reducción de la vulnerabilidad, significa sin duda apostar por una revisión de los valores sociales imperantes. En las actuales circunstancias, el mero hecho de estar conectado no implica precisamente evitar la exclusión, sino que el riesgo de vulnerabilidad aumenta exponencialmente al tiempo de conexión y a la calidad de los intercambios que se producen, algo difícil de evaluar.

El olvido, consciente y deliberado, de la escuela en la mayoría de muchas propuestas de acercamiento de los jóvenes a la cibercultura, más cercanas al mundo de la educación no formal, puede significar precisamente intentar desvincular este proceso de lo programático que significa la escuela y apostar por modelos fidelizadores más allá de la escuela. Así, siguiendo a Caledoni (2002), lo que se plantea es un cambio de registro a distintos niveles:

· en la misma definición de escuela, de docente y de discente;

- en el acomodo y apoyo que requieren docentes y equipos directivos;

- en la incardinación de la escuela en su entorno.

Los aspectos mencionados refieren a la necesidad de establecer modelos escolares más acordes con los tiempos en que vivimos. La escuela decimonónica que todavía conocemos debe ser revisada a fondo y la oportunidad de oro que nos plantea la sociedad del conocimiento debe ser sabiamente encajada. Una institución, como la escuela, creada para iluminar y diseminar conocimiento no puede quedarse al margen de este desafío.

En este sentido, como venimos insistiendo, más que rediseñar el desarrollo instructivo de las secuencias didácticas, que sin duda es una tarea inaplazable, lo que debemos reforzar son las capacidades mediante las cuales el sujeto se encara con este nuevo mundo. Así, los mecanismos de autorregulación y de corregulación son totalmente necesarios: la capacidad del sujeto para autoestablecer los límites de uso de la nueva herramienta se convierte en un objetivo educativo de primer orden; pero además, también es necesario acordar dispositivos que permitan regular externamente ese uso, de manera que el sujeto se someta a una heteroevaluación por parte de quien ostente la autoridad moral para ese sujeto, sean progenitores, profesorado o incluso iguales. El papel de la familia, para la mayoría de los adolescentes, parece crucial en este sentido, pero también el del círculo de amistades y por supuesto el papel del 
profesorado.

\section{Bibliografía}

BERLAND et al. (2001) Proceed with Caution: A Report on the Quality of Health Information on the Internet. California HealthCare Foundation, Report Summary and Complete Study [citado por Vivas; Armayones et al, 2004].

CALEDONI, Paolo (2002) “Dirigenti scolastici e nuove tecnologie”, en Teoría de la Educación: Educación y Cultura en la Sociedad de la Información. vol. 3; http://www3.usal.es/ teoriaeducacion/rev_numero_03/n3_art_calidoni.htm [11.12.2004]

CASTELLS, Manuel; DÍAZ DE ISLA, Maria Isabel (2001) Diffusion and uses of Internet in Catalonia and in Spain. A Commented Summary of Available Evidences, as of 2001. www.uoc.edu/in3/wp/picwp1201 [11.12.2004]

CONDE, H.; MUÑOZ, J. M. (2002) "Políticas comunitarias de juventud: el factor territorial en la sociedad global”, en Teoría de la Educación: Educación y Cultura en la Sociedad de la Información, vol. 3; http://www3.usal.es/ teoriaeducacion/rev_numero_03/n3_art_valdivieso-rodriguez.htm [11.12.2004]

EDUTEKA (2004) "Valores ciudadanos que las TIC pueden promover”, www.eduteka.org [última consulta: 14.11.2004]

GARCÍA CANCLINI, Néstor (2004) Diferentes, desiguales y desconectados. Barcelona, Gedisa.

MIZRACH, Steve (s.d.) "Lost in Cyberspace: A Cultural Geography of Cyberspace”, en http://www.fiu.edu/ mizrachs/lost-in-cyberspace.html [21.11.2004]

NOGUERA, Elena (2004) “Els valors a Internet” en http://www.callusdigital.org/lacenet/guiaeducativa/docs/pdf/valors_enoguera.pdf [15.10.2004]

PRATS, Enric [Coord.] (2004) Ética de la información. Barcelona, Ediuoc.

UNICEF (2003) Informe mundial de la infancia 2004. Ginebra: Unicef.

VIVAS, Pep; ARMAYONES, Manuel et al (2004) "Salut i Internet: cerca, temàtica i qualitat del seu contingut”, en www.uoc.edu/in3/dt/20375/index.html [8.11.2004]

YUS, Francisco (2001) "Ciberpragmática. Entre la compensación y el desconcierto". en http://www.cibersociedad.net/archivo/articulo.php?art=42 [30.9.2004]

\section{Notas:}

[1] Esta reflexión inicial tiene una alta carga pedagógica cuando reparamos en el principio educativo de la contextualización, algo que Internet ha conseguido romper, como veremos más adelante.

[2] http://www.cisco.com/global/MX/sne/pc/industria/educacion/index.shtml [12.11.2004]

[3] Sitio web de la Fundación Gabriel Piedrahita Uribe para jóvenes de educación secundaria: www.eduteka.org

[4] "La significación de unos datos no proviene de la capacidad técnica para organizarlos de una manera determinada y producir información, sino de la intencionalidad y, por lo tanto, de las categorías previas o prejuicios con que el profesional gestiona la información.” Prats, 2004, 112.

[5] "Las jergas sirven para un importante propósito dual: por un lado, determinar el derecho del usuario a pertenecer al grupo que usa la jerga y, por otro lado, generar fronteras de uso discursivo con otros grupos 
de hablantes con los que el ser humano no desea ser confundido”. Yus, 2001

[6] Este estudio propone una serie de medidas para aumentar la validez de la información de los sitios web sobre temas de salud; en sus conclusiones cabe destacar el énfasis en que los diseñadores e informantes de estos sitios conozcan las características de los demandantes de información para responder mejor a sus necesidades, algo que podría limitar con la confidencialidad de datos. Ver Prats (2004).

[7] Casi lo mismo se está anunciando para la tecnología digital terrestre en el campo televisivo (TDT); los defensores de la telebasura argumentan que la TDT incrementará la oferta de programas lo cual redundará en la democratización audiovisual reduciendo los supuestos efectos nocivos de la actual programación.

Como suele acontecer, más oferta no tiene por qué ir acompañada de más calidad y lo que puede pasar es que tengamos más telebasura por centímetro cúbico de espacio audiovisual.

[8] No nos hemos atrevido a corroborar si esas previsiones se han cumplido, pero mucho nos tememos que estamos bastante alejados de esas cifras. Pero en el feliz caso de que estuviéramos en ese nivel de ciberconexión, lo alarmante sería que hemos perdido cinco años sin formar adecuadamente a esos jóvenes.

[9] A la espera de una universalización real de los sistemas de cable e inalámbricos, no hay que olvidar que las redes de Internet sólo son funcionales “encima” de redes conmutadas de línea telefónica, por lo que la existencia de esta última es condición imprescindible para la extensión de la primera.

[10] www.cnice.mec.es [12.11.2004]

[11] http://www.euia.org/joves/noves_tecnologies.htm, en el sitio web de Esquerra Unida i Alternativa, equivalencia en Catalunya de Izquierda Unidad, que acoge el programa

[12] En esta misma monografía digital se presentan algunas, de las ya innumerables, acciones y experiencias con y para jóvenes.

[13] Por miedo a ser foco de un ataque soviético, Arpanet (desarrollada por el Pentágono en 1969) pretendía descentralizar la conmutación de datos mediante una red alternativa que supliera el supuesto destrozo de las líneas telefónicas. No desapareció hasta 1990, relevada por la World Wide Web, producto de Timothy Berners-Lee, investigador del CERN de Ginebra, con fines académicos y científicos. Como se sabe, en la última década se ha añadido el sector comercial, siempre más cauto y reservado en esto de incorporar demasiados cambios.

[14] Relativo a esa "química” que se da entre sujetos cuando se relacionan, estableciendo una suerte de intercambio hormonal también en términos estrictamente biológicos.

(C) Ediciones Universidad de Salamanca

Webmasters del volumen actual: Francisco Ignacio Revuelta Domínguez, Lourdes Pérez Sánchez

Correos electrónicos por orden: fird@usal.es

o bien lopesan@usal.es 